\title{
High-static-low-dynamic-stiffness vibration isolation enhanced by damping nonlinearity
}

\author{
LU ZeQi ${ }^{1,4}$, BRENNAN Michael ${ }^{2}$, DING $\mathrm{Hu}^{1,4}$ \& CHEN LiQun ${ }^{1,3,4^{*}}$ \\ ${ }^{1}$ Shanghai Institute of Applied Mathematics and Mechanics, Shanghai University, Shanghai 200072, China; \\ ${ }^{2}$ Departamento de Engenharia Mecânica, Universidade Estadual Paulista (UNESP), Ilha Solteira (SP) 56-15385-000, Brasil; \\ ${ }^{3}$ Department of Mechanics, Shanghai University, Shanghai 200444, China; \\ ${ }^{4}$ Shanghai Key Laboratory of Mechanics in Energy Engineering, Shanghai University, Shanghai 200072, China
}

Received November 8, 2017; accepted May 14, 2018; accepted September 3, 2018

\begin{abstract}
High-static-low-dynamic-stiffness (HSLDS) nonlinear isolators have proven to have an advantage over linear isolators, because HSLDS nonlinear isolators allow low-frequency vibration isolation without compromising the static stiffness. Previously, these isolators have generally been assumed to have linear viscous damping, degrading the performance of the isolator at high frequencies. An alternative is to use nonlinear damping, where the nonlinear behavior is achieved by configuring linear dampers so they are orthogonally aligned to the excitation direction. This report compares the performances of single-stage and two-stage isolators with this type of damping with the corresponding isolators containing only linear viscous damping. The results show that both isolators with linear viscous damping and nonlinear damping reduce the transmissibility around the resonance frequencies, but the results show that the isolators with nonlinear damping perform better at high frequencies.
\end{abstract}

nonlinear vibration isolation, transmissibility, high-static-low-dynamic-stiffness, damping nonlinearity

Citation: $\quad$ Lu Z Q, Brennan M, Ding H, et al. High-static-low-dynamic-stiffness vibration isolation enhanced by damping nonlinearity. Sci China Tech Sci, 2018, 61, https://doi.org/10.1007/s11431-017-9281-9

\section{Introduction}

One way to improve low-frequency vibration isolation without having excessive static deflection is to use a nonlinear isolator [1]. A simple way to model this type of isolator is to combine lateral linear springs with a linear (vertical) spring orthogonal to the lateral springs [2-5], resulting in an isolator that has high-static-low-dynamic-stiffness (HSLDS), and many studies have shown there are many ways to achieve this characteristic [1,6-11]. Recently, this concept has been further developed as a two-stage isolator [12-14]. In all these cases, the damping was due to a linear viscous damper in parallel with the vertical springs.

Damping nonlinearity has also been widely investigated in

* Corresponding author (email: 1qchen@staff.shu.edu.cn) the context of vibration isolation, for example [15-22]. One type of damping where the damping force is proportional to the cube of the velocity has been studied by several researchers [19-22]. The studies have shown that this type of damping has some advantages compared to linear viscous damping in overcoming the compromise between suppressing the response at the resonance frequency and maintaining good performance at high frequency. Ho et al. [23] studied a single-degree-of-freedom nonlinear vibration isolation system with both stiffness and cubic damping nonlinearity. They used relatively simple analytical models to characterize the system in terms of power flow, using output frequency response functions (OFRFs). Recently, the effects of lateral dampers, which offer some advantages compared to vertical damping, have been considered in combination with a vertical linear spring $[24,25]$. The studies showed that this ar- 
rangement has the advantage of improving the performance at the resonance frequency without degrading the isolation performance at high frequencies for both force and displacement excited systems, provided that the level of excitation is not too large.

This study investigates the differences between having linear or nonlinear damping in an HSLDS vibration isolation system. The two damping configurations are (1) a linear viscous damper in parallel with the vertical spring and (2) lateral linear viscous dampers in parallel with the lateral springs (in this case, the nonlinear damping is due to the geometry). The aspiration is that the merits of both mechanisms are combined by including both nonlinear stiffness and nonlinear damping. The frequency range of vibration should be increased, and the response around resonance and the high-frequency vibration isolation should be improved. Furthermore, a single-stage isolator and a two-stage isolator are considered.

\section{Single stage isolator}

Figure 1 shows a lumped parameter model of a vibration isolation system with stiffness and damping nonlinearity for a suspended mass $m$. The stiffness nonlinearity is represented by the horizontal spring $k_{\mathrm{h}}$ and the vertical spring $k_{\mathrm{v}}$. Two cases are compared: (1) when the system only has linear viscous damping due to the vertical damper $c_{\mathrm{v}}$ and (2) when the system only has nonlinear dampers $c_{\mathrm{h}}$. If the mass is excited with a harmonic force $f_{\mathrm{e}}(t)=F_{\mathrm{e}} \cos (\omega t)$, the equation of motion for the system is given by

$m x^{\prime \prime}+c_{\mathrm{v}} x^{\prime}+c_{\mathrm{n}}(x) x^{\prime}+k_{\mathrm{n}}(x) x=F_{\mathrm{e}} \cos (\omega t)$,

where the nonlinear damping coefficient is given by $c_{\mathrm{n}}(x)$ $=2 c_{\mathrm{h}} x^{2} /\left(x^{2}+l^{2}\right)$ and the nonlinear stiffness term, which includes the vertical and horizontal stiffness, is given by $k_{\mathrm{n}}(x)$ $=k_{\mathrm{v}}+2 k_{\mathrm{h}}\left[1-l_{o} /\left(x^{2}+l^{2}\right)^{1 / 2}\right]$, where $l$ represents the dimension shown in Figure 1. Although eq. (1) has been written with

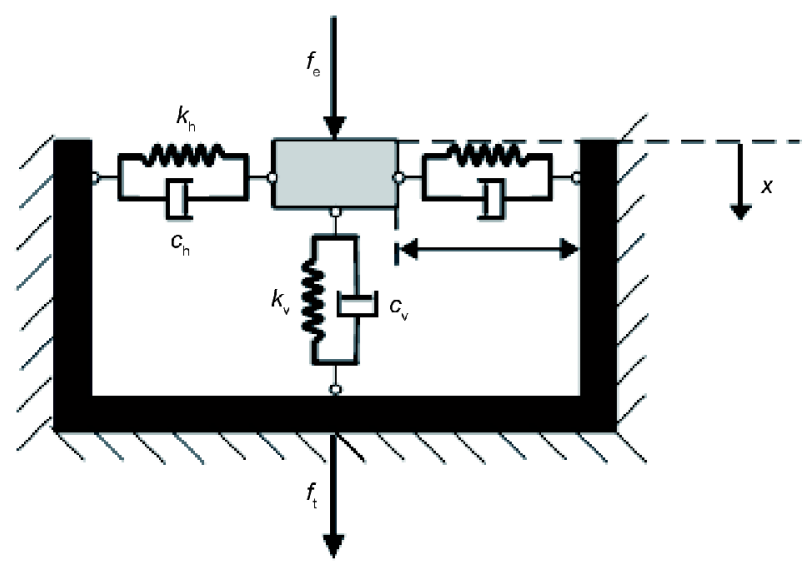

Figure 1 (Color online) Model of a single-stage HSLDS isolator. both linear and nonlinear damping terms for conciseness, these terms are also separately considered in the simulations presented later to compare their effects. Eq. (1) can be written in a non-dimensional form as

$\widehat{x}^{\prime \prime}+2 \zeta_{\mathrm{v}} \widehat{x}^{\prime}+2 \zeta_{\mathrm{n}}(\widehat{x}) \hat{x}^{\prime}+\widehat{k}_{\mathrm{n}}(\widehat{x}) \widehat{x}=\widehat{F}_{\mathrm{e}} \cos (\Omega \tau)$,

where $\quad \zeta_{\mathrm{n}}(\widehat{x})=2 \zeta_{\mathrm{h}} \widehat{x}^{2} /\left(\widehat{x}^{2}+1\right) \quad$ and $\quad \widehat{k}_{\mathrm{n}}(\widehat{x})=1+2 \widehat{k}$ $\cdot\left[1-1 / \widehat{l}\left(\widehat{x}^{2}+1\right)^{1 / 2}\right] ; \widehat{x}=x / l, \widehat{l}=l / l_{\mathrm{o}}$, in which $l_{\mathrm{o}}$ represents the free length of the horizontal springs, $\widehat{k}=k_{\mathrm{h}} / k_{\mathrm{v}}$, $\zeta_{\mathrm{v}}=c_{\mathrm{v}} /\left(2 m \omega_{\mathrm{n}}\right), \zeta_{\mathrm{h}}=c_{\mathrm{h}} /\left(2 m \omega_{\mathrm{n}}\right)$, in which $\omega_{\mathrm{n}}=\left(k_{\mathrm{v}} / m\right)^{1 / 2}$, $\widehat{F_{\mathrm{e}}}=F_{\mathrm{e}} / k_{\mathrm{v}} l, \Omega=\omega / \omega_{\mathrm{n}}, \tau=\omega_{\mathrm{n}} t$. If $\widehat{k}=1$, then $\hat{l} \geq 2 / 3$ to ensure that the stiffness of the isolator does not become negative and have a snap-through characteristic.

For small displacements compared to the length $l$, eq. (2) can be written as a Duffing equation in which the nonlinear damping and stiffness terms in eq. (2) become $\zeta_{\mathrm{n}}(\widehat{x}) \approx 2 \zeta_{\mathrm{h}} \widehat{x}^{2}$ and $k_{\mathrm{n}}(\widehat{x}) \approx\left(\alpha+\gamma \widehat{x}^{2}\right)$, respectively, where $\alpha=1-2 \widehat{k}$ $(1-\hat{l}) / \hat{l}$ and $\gamma=\widehat{k} / \hat{l}$. The nonlinear damping is proportional to the square of the displacement, so the damping is small for small amplitudes of displacement and large for large amplitudes of displacement. The actual and approximate stiffness and damping terms are plotted in Figure 2(a) and (b), respectively, for $\widehat{k}=1$ and $\widehat{l}=0.7$. The figure shows that the actual and approximate stiffness and damping terms are similar for non-dimensional displacements, because the difference is less than 0.2 . In the following analysis, the small displacement (less than 0.2 ) approximations are valid. This is not a severe restriction, as the displacements of a practical system are unlikely to be $20 \%$ of the length of the horizontal spring or damper. It is also assumed that the maximum value of the excitation force is such that analytical results are valid $[4,24]$.

Applying the harmonic balance method [26] and assuming a solution of the form $\widehat{x}(\tau)=\bar{X} \cos (\Omega \tau+\phi)$ yields

$$
\begin{aligned}
& \left(\alpha-\Omega^{2}\right) \widehat{X}+\frac{3}{4} \gamma \widehat{X}^{3}=\widehat{F_{\mathrm{e}}} \cos \phi, \\
& \zeta_{\mathrm{v}} \Omega \widehat{X}+\frac{1}{4} \zeta_{\mathrm{h}} \Omega \widehat{X}^{3}=\widehat{F_{\mathrm{e}}} \sin \phi,
\end{aligned}
$$

where the terms containing $\cos (3 \Omega \tau)$ or $\sin (3 \Omega \tau)$ are neglected. The amplitude-frequency relationship can be derived from eqs. (3) and (4) as

$\left(\left(\alpha-\Omega^{2}\right) \widehat{X}+\frac{3}{4} \gamma \widehat{X}^{3}\right)^{2}+\left(2 \zeta_{\mathrm{v}} \Omega \widehat{X}+\frac{1}{2} \zeta_{\mathrm{h}} \Omega \widehat{X}^{3}\right)^{2}=\widehat{F}_{\mathrm{e}}^{2}$.

This is a quadratic equation in $\Omega^{2}$ that can be solved to yield

$\Omega_{1,2}=\left\{\alpha+\frac{3}{4} \gamma \widehat{X}^{2}-\frac{1}{2}\left(2 \zeta_{\mathrm{v}}+\frac{1}{2} \zeta_{\mathrm{h}} \widehat{X}^{2}\right)^{2}\right.$ 

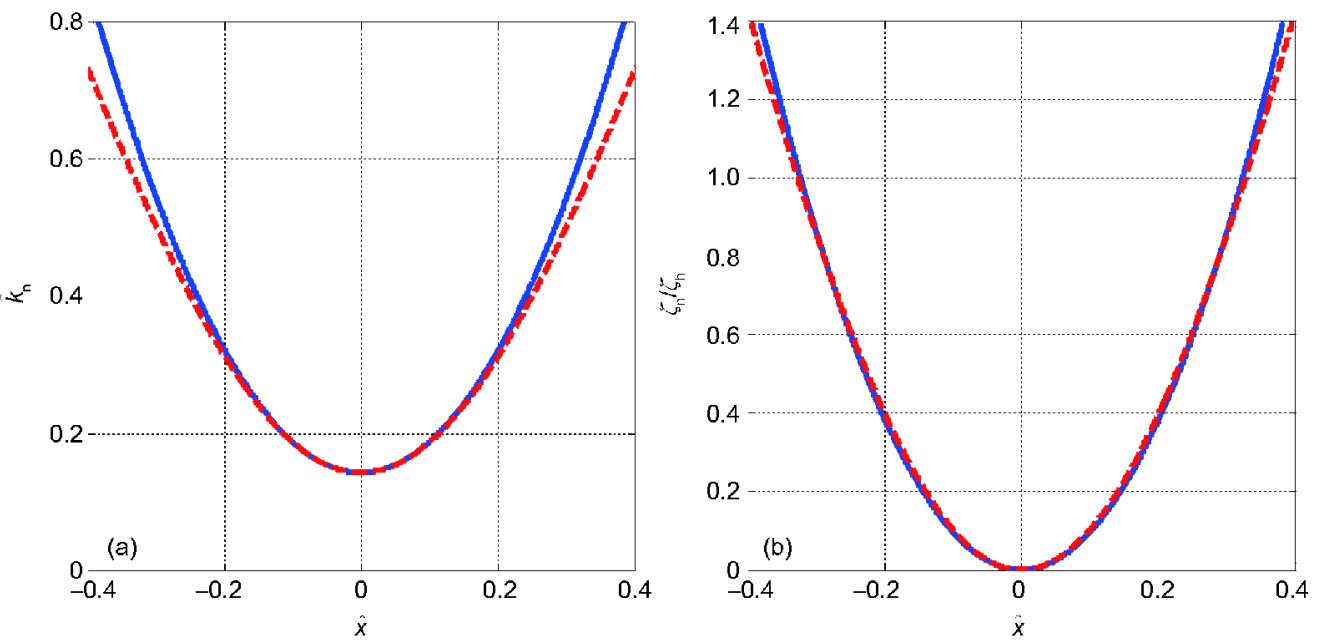

Figure 2 (Color online) Nonlinear parameters for the single-stage isolator for $\widehat{k}=1, \hat{l}=0.7$, solid line: exact results; dashed line approximate results; (a) nonlinear stiffness (including the vertical stiffness); (b) normalized nonlinear damping ratio.

$\pm \frac{1}{\widehat{X}}\left[\widehat{F}_{\mathrm{e}}^{2}+\frac{1}{4}\left(\zeta_{\mathrm{v}}+\frac{1}{4} \zeta_{\mathrm{h}} \widehat{X}^{2}\right)^{4} \widehat{X}^{2}\right.$

$\left.\left.-\left(\zeta_{\mathrm{v}}+\frac{1}{4} \zeta_{\mathrm{h}} \widehat{X}^{2}\right)^{2}\left(\alpha \widehat{X}^{2}+\frac{3}{4} \gamma \widehat{X}^{4}\right)\right]^{1 / 2}\right\}^{1 / 2}$,

which are the two stable branches (called the resonance and non-resonance branches) of the frequency response curve.

The non-dimensional transmitted force through the springs and the dampers of the isolator, and the force is provided by $\widehat{f_{\mathrm{t}}}=2 \zeta_{\mathrm{v}} \widehat{x}^{\prime}+2 \zeta_{\mathrm{h}} \widehat{x}^{2} \widehat{x}^{\prime}+\alpha \widehat{x}+\gamma \widehat{x}^{3}$.

The transmitted force has the form $\widehat{F_{\mathrm{t}}} \cos \left(\Omega \tau+\varphi_{T}\right)$, where

$\widehat{F_{\mathrm{t}}}=\widehat{X}\left[\left(\alpha+\frac{3}{4} \gamma \widehat{X}^{2}\right)^{2}+\Omega^{2}\left(2 \zeta_{\mathrm{v}}+\frac{1}{2} \zeta_{\mathrm{n}} \widehat{X}^{2}\right)^{2}\right]^{1 / 2}$.

The modulus of force transmissibility is determined by $\left|T_{F}\right|=\widehat{F_{\mathrm{t}}} / \widehat{F_{\mathrm{e}}}$.

Thus, the magnitude of the transmissibility for the resonance and non-resonance branches can be determined by substituting the two solutions for $\Omega_{1,2}$ in eq. (6) into eq. (8) and combining with eq. (9) to give

$\left|T_{F}\right|_{1}=\frac{\widehat{X}\left[\left(\alpha+\frac{3}{4} \gamma \widehat{X}^{2}\right)^{2}+\Omega_{1}^{2}\left(2 \zeta_{\mathrm{v}}+\frac{1}{2} \zeta_{\mathrm{h}} \widehat{X}^{2}\right)^{2}\right]^{1 / 2}}{\hat{F}_{\mathrm{e}}}$

$\left|T_{F}\right|_{2}=\frac{\widehat{X}\left[\left(\alpha+\frac{3}{4} \gamma \widehat{X}^{2}\right)^{2}+\Omega_{2}^{2}\left(2 \zeta_{\mathrm{v}}+\frac{1}{2} \zeta_{\mathrm{h}} \widehat{X}^{2}\right)^{2}\right]^{1 / 2}}{\hat{F}_{\mathrm{e}}}$.

The peak transmissibility corresponds to the peak displacement response, which can be determined by noting that eq. (6) are equal at this frequency; hence,

$$
\begin{aligned}
& \widehat{F}_{\mathrm{e}}^{2}+\frac{1}{4}\left(\zeta_{\mathrm{v}}+\frac{1}{4} \zeta_{\mathrm{h}} \widehat{X}^{2}\right)^{4} \widehat{X}^{2} \\
& -\left(\zeta_{\mathrm{v}}+\frac{1}{4} \zeta_{\mathrm{h}} \widehat{X}^{2}\right)^{2}\left(\alpha \bar{X}^{2}+\frac{3}{4} \gamma \widehat{X}^{4}\right)=0 .
\end{aligned}
$$

The solution to eq. (11) gives

$$
\begin{aligned}
\widehat{X}_{\max }= & \left\{\left[-\frac{1}{4} \zeta_{\mathrm{v}}^{4}+\alpha \zeta_{\mathrm{v}}^{2}+\left[\left(\frac{1}{4} \zeta_{\mathrm{v}}^{4}-\alpha \zeta_{\mathrm{v}}^{2}\right)^{2}\right.\right.\right. \\
& \left.\left.-\left(\zeta_{\mathrm{v}}^{3} \zeta_{\mathrm{h}}-3 \gamma \zeta_{\mathrm{v}}^{2}-2 \alpha \zeta_{\mathrm{v}} \zeta_{\mathrm{h}}\right) \hat{F}_{\mathrm{e}}^{2}\right]^{1 / 2}\right] \\
& \left./\left(\frac{1}{2} \zeta_{\mathrm{v}}^{3} \zeta_{\mathrm{h}}-\frac{3}{2} \gamma \zeta_{\mathrm{v}}^{2}-\alpha \zeta_{\mathrm{v}} \zeta_{\mathrm{h}}\right)^{1 / 2}\right\}
\end{aligned}
$$

The frequency at the maximum transmissibility can be derived by substituting eq. (12) into eq. (6) to provide

$$
\begin{aligned}
\Omega_{F \max }= & \left\{\frac{1}{2}\left[\alpha+\left(\alpha^{2}+\frac{3 \gamma \widehat{F}_{\mathrm{e}}^{2}}{\zeta_{\mathrm{v}}^{2}}\right)^{1 / 2}\right]\right. \\
& \left.-\frac{1}{2}\left\{2 \zeta_{\mathrm{v}}+\zeta_{\mathrm{h}} \frac{1}{3 \gamma}\left[-\alpha+\left(\alpha^{2}+\frac{3 \gamma \widehat{F}_{\mathrm{e}}^{2}}{\zeta_{\mathrm{v}}^{2}}\right)^{1 / 2}\right]^{2}\right]\right\}^{1 / 2} .
\end{aligned}
$$

The maximum force that can be applied so the peak in the response occurs at $\Omega=1$ can be determined by rearranging eq. (13) to provide

$$
\begin{aligned}
\widehat{F}_{\max }= & \left\{\left(\frac{1}{4} \zeta_{\mathrm{v}}^{4}-\alpha \zeta_{\mathrm{v}}^{2}\right)^{2}-\left[\frac{4}{3 \gamma}(1-\alpha)\right.\right. \\
& \left.\times\left(\frac{1}{2} \zeta_{\mathrm{v}}^{3} \zeta_{\mathrm{h}}-\frac{3}{2} \gamma \zeta_{\mathrm{v}}^{2}-\alpha \zeta_{\mathrm{v}} \zeta_{\mathrm{h}}\right)+\frac{1}{4} \zeta_{\mathrm{v}}^{4}-\alpha \zeta_{\mathrm{v}}^{2}\right]^{2} \\
& \left./\left(\zeta_{\mathrm{v}}^{3} \zeta_{\mathrm{h}}-3 \gamma \zeta_{\mathrm{v}}^{2}-2 \alpha \zeta_{\mathrm{v}} \zeta_{\mathrm{h}}\right)\right\}^{1 / 2}
\end{aligned}
$$


where $\widehat{F}_{\max }$ is similar to the case when the isolator has a linear rather than a nonlinear damping, which is discussed in ref. [4]. The mathematical details for $\widehat{F}_{\max }$ are given as follows when the horizontal dampers are removed [4]

$\widehat{F}_{\max }=\left\{\frac{4 \zeta_{\mathrm{v}}^{2}}{3 \gamma}\left[\left(2+3 \zeta_{\mathrm{v}}^{2}-\alpha\right)^{2}-\zeta_{\mathrm{v}}^{4}-\alpha\left(\alpha-2 \zeta_{\mathrm{v}}^{2}\right)\right]\right\}^{1 / 2}$.

To investigate whether it is better to place the damper in parallel with the vertical spring or if it is better to place dampers in parallel with the horizontal springs, the force transmissibilities for both cases are plotted in Figure 3. Also shown for comparison is a faint grey line that represents the transmissibility of the system with the horizontal springs and dampers removed and with $\zeta_{\mathrm{v}}=0.005$. For these simulations, the amplitude of the excitation force is set so that $\widehat{F_{\mathrm{e}}}=0.1 \widehat{F}_{\text {max }}=0.0022$, where $\widehat{F}_{\text {max }}$ represents the force that causes the jump-down frequency to occur at $\Omega=1$ when the linear damping ratio is set to $\zeta_{\mathrm{v}}=0.05$. Figure 3(a) shows that the vertical damper is effective in reducing the response around the resonance frequency, but the vertical damper degrades the vibration performance at high frequencies. In addition, Figure 3(b) shows that the horizontal dampers are not as effective around the resonance frequency, but they do not degrade the isolator performance at high frequencies in the isolation region. Higher damping could, however, improve the performance at resonance. Thus, horizontal dampers offer a clear advantage compared to vertical dampers for the low level of excitation considered. At higher amplitudes of excitation or with higher damping, the horizontal dampers may generate undesirable harmonics, as discussed in ref. [25].

\section{Two-stage vibration isolation system}

\subsection{Force transmissibility}

Figure 4 shows a lumped parameter model of two-stage nonlinear vibration isolation system. It consists of the suspended mass, $m_{1}$, and an intermediate mass, $m_{2}$. The upper stage has horizontal springs, $k_{\mathrm{h} 1}$, a vertical spring, $k_{\mathrm{v} l}$, a horizontal linear viscous damper, $c_{\mathrm{h} 1}$, and a vertical linear viscous damper, $c_{\mathrm{v} v}$. The lower stage also has horizontal and vertical springs and lower horizontal and vertical linear viscous dampers. With the single-stage isolator, the vertical and horizontal stiffness elements are considered together, but the horizontal and vertical dampers are separately considered.

The system is excited harmonically with force $f_{\mathrm{e}}(t)=$ $F_{\mathrm{e}} \cos (\omega t)$, and the matrix equation of the motion is given by

$\mathbf{M x} "+\mathbf{C}(\mathbf{x}) \mathbf{x}^{\prime}+\mathbf{K}(\mathbf{x}) \mathbf{x}=\mathbf{f}$,

where $\mathbf{M}=\left[\begin{array}{cc}m_{1} & 0 \\ 0 & m_{2}\end{array}\right], \mathbf{x}=\left\{\begin{array}{l}x_{1} \\ x_{2}\end{array}\right\}, \mathbf{f}=\left\{\begin{array}{c}F_{\mathrm{e}} \cos (\omega t) \\ 0\end{array}\right\}$,

$\mathbf{C}(x)=$

$\left[\begin{array}{cc}c_{\mathrm{v} 1}+\frac{2 c_{\mathrm{h} 1}\left(x_{1}-x_{2}\right)^{2}}{l_{1}^{2}+\left(x_{1}-x_{2}\right)^{2}} & -c_{\mathrm{v} 1}-\frac{2 c_{\mathrm{h} 1}\left(x_{1}-x_{2}\right)^{2}}{l_{1}^{2}+\left(x_{1}-x_{2}\right)^{2}} \\ -c_{\mathrm{v} 1}-\frac{2 c_{\mathrm{h} 1}\left(x_{1}-x_{2}\right)^{2}}{l_{1}^{2}+\left(x_{1}-x_{2}\right)^{2}} & c_{\mathrm{v} 1}+c_{\mathrm{v} 2}+\frac{2 c_{\mathrm{h} 1}\left(x_{1}-x_{2}\right)^{2}}{l_{1}^{2}+\left(x_{1}-x_{2}\right)^{2}}+\frac{2 c_{\mathrm{h} 2} x_{2}^{2}}{l_{2}^{2}+x_{2}^{2}}\end{array}\right]$,

$\mathbf{K}(x)=\left[\begin{array}{l}k_{\mathrm{v} 1}+2 k_{\mathrm{h} 1}\left(1-\frac{l_{01}}{\sqrt{\left(x_{1}-x_{2}\right)^{2}+l_{1}^{2}}}\right) \\ -k_{\mathrm{v} 1}-2 k_{\mathrm{h} 1}\left(1-\frac{l_{01}}{\sqrt{\left(x_{1}-x_{2}\right)^{2}+l_{1}^{2}}}\right)\end{array}\right.$

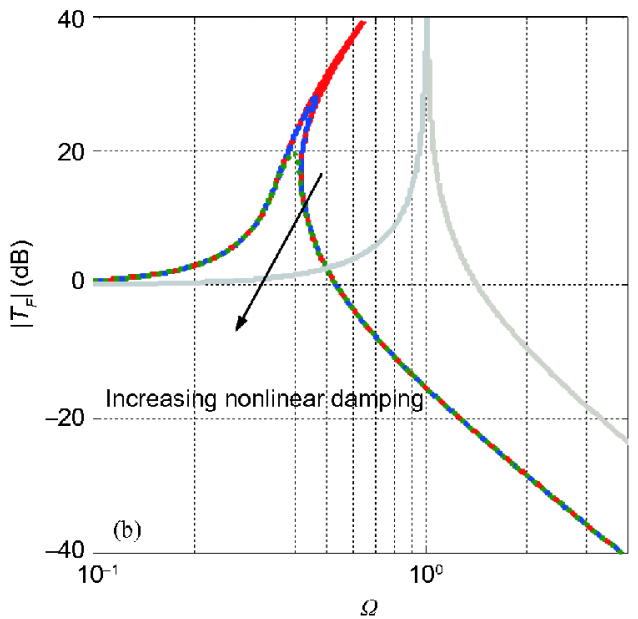

Figure 3 (Color online) Comparison of the effects of linear and nonlinear damping on the force transmissibility for high-static-low-dynamic-stiffness isolation, for $\widehat{k}=1, \widehat{F}_{\mathrm{e}}=0.1 \widehat{F}_{\max }=0.0022$, and $\widehat{l}=0.7$; (a) horizontal damping $\zeta_{\mathrm{h}}=0$ and vertical damping is changed; solid line, $\zeta=0.005$; dashed line, $\zeta_{\mathrm{v}}=0.05$; and dotted line, $\zeta_{\mathrm{v}}=0.1 ;$ (b) vertical damping $\zeta_{\mathrm{v}}=0$ and horizontal damping is changed; solid line, $\zeta_{\mathrm{h}}=0.05 ;$ dashed line, $\zeta_{\mathrm{h}}=0.5$; and dotted line, $\zeta_{\mathrm{h}}=1$. The faint grey line represents the linear isolator that only has vertical stiffness and damping with $\zeta_{\mathrm{v}}=0.005$. 


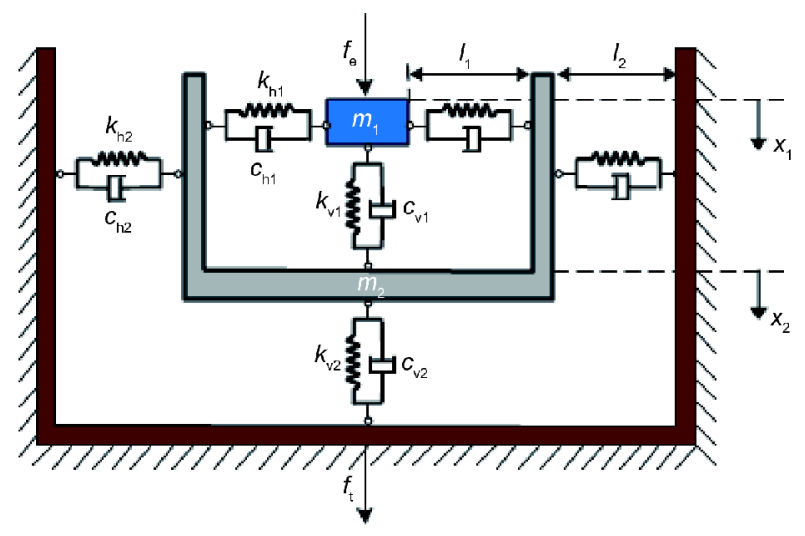

Figure 4 (Color online) Model of a two-stage HSLDS isolator.

$-k_{\mathrm{v} 1}-2 k_{\mathrm{h} 1}\left(1-\frac{l_{01}}{\sqrt{\left(x_{1}-x_{2}\right)^{2}+l_{1}^{2}}}\right)$

$\left.k_{\mathrm{v} 1}+2 k_{\mathrm{h} 1}\left(1-\frac{l_{01}}{\sqrt{\left(x_{1}-x_{2}\right)^{2}+l_{1}^{2}}}\right)+k_{\mathrm{v} 2}+2 k_{\mathrm{h} 2}\left(1-\frac{l_{02}}{\sqrt{x_{2}^{2}+l_{2}^{2}}}\right)\right)$

and $l_{01}$ and $l_{02}$ represent the initial lengths of the horizontal springs, and $l_{1}$ and $l_{2}$ represent their lengths when they are in the horizontal position in upper and lower stages, respectively. With the single-stage isolator, approximations are made for small amplitudes. Also, setting $l_{1}=l_{2}$, eq. (16) can be written in a non-dimensional form as

$\widehat{\mathbf{M}} \widehat{\mathbf{x}}^{\prime \prime}+\widehat{\mathbf{C}}_{\mathrm{v}} \widehat{\mathbf{x}}^{\prime}+\widehat{\mathbf{C}}_{\mathrm{n}}(\widehat{\mathbf{x}}) \widehat{\mathbf{x}}^{\prime}+\widehat{\mathbf{K}}(\widehat{\mathbf{x}}) \widehat{\mathbf{x}}=\hat{\mathbf{f}}$,

where $\widehat{\mathbf{M}}=\left[\begin{array}{ll}1 & 0 \\ 0 & \mu\end{array}\right], \widehat{\mathbf{x}}=\left\{\begin{array}{l}\widehat{x}_{1} \\ \widehat{x}_{2}\end{array}\right\}, \hat{\mathbf{f}}=\left\{\begin{array}{c}\widehat{F_{\mathrm{e}}} \cos (\Omega \tau) \\ 0\end{array}\right\}$,

$\widehat{\mathbf{K}}(\widehat{x})=$

$\left[\begin{array}{cc}\Omega_{1}^{2}+\gamma_{1}\left(\widehat{x}_{1}-\widehat{x}_{2}\right)^{2} & -\Omega_{1}^{2}-\gamma_{1}\left(\widehat{x}_{1}-\widehat{x}_{2}\right)^{2} \\ -\Omega_{1}^{2}-\gamma_{1}\left(\widehat{x}_{1}-\widehat{x}_{2}\right)^{2} & \Omega_{1}^{2}+\Omega_{2}^{2}+\gamma_{1}\left(\widehat{x}_{1}-\widehat{x}_{2}\right)^{2}+\gamma_{2} \widehat{x}_{2}^{2}\end{array}\right]$,

$\widehat{\mathbf{C}}_{\mathrm{n}}(\widehat{x})=2\left[\begin{array}{cc}\zeta_{\mathrm{n} 1}\left(\widehat{x}_{1}-\widehat{x}_{2}\right)^{2} & -\zeta_{\mathrm{n} 1}\left(\widehat{x}_{1}-\widehat{x}_{2}\right)^{2} \\ -\zeta_{\mathrm{n} 1}\left(\widehat{x}_{1}-\widehat{x}_{2}\right)^{2} & \zeta_{\mathrm{n} 1}\left(\widehat{x}_{1}-\widehat{x}_{2}\right)^{2}+\zeta_{\mathrm{n}} \widehat{x}_{2}^{2}\end{array}\right]$,

$\widehat{\mathbf{C}}_{\mathrm{v}}=2\left[\begin{array}{cc}\zeta_{\mathrm{v} 1} & -\zeta_{\mathrm{v} 1} \\ -\zeta_{\mathrm{v} 1} & \zeta_{\mathrm{v} 1}+\zeta_{\mathrm{v} 2}\end{array}\right], \zeta_{\mathrm{v} 1}=c_{\mathrm{v} 1} /\left(2 m_{1} \omega_{\mathrm{n}}\right)$,

$\zeta_{\mathrm{h} 1}=c_{\mathrm{h} 1} /\left(2 m_{1} \omega_{\mathrm{n}}\right), \zeta_{\mathrm{n} 1}=\zeta_{\mathrm{h} 1}\left(1-\widehat{l}^{2}\right) / \widehat{l}^{2}$,

$\zeta_{\mathrm{v} 2}=c_{\mathrm{v} 2} /\left(2 m_{1} \omega_{\mathrm{n}}\right), \zeta_{\mathrm{h} 2}=c_{\mathrm{h} 2} /\left(2 m_{1} \omega_{\mathrm{n}}\right)$,

$\zeta_{\mathrm{n} 2}=\zeta_{\mathrm{h} 2}\left(1-\widehat{l}^{2}\right) / \widehat{l}^{2}, \Omega_{1}^{2}=1-2 \widehat{k}_{\mathrm{h} 1}(1-\widehat{l}) / \widehat{l}$,

$\Omega_{2}^{2}=1+\widehat{k}_{\mathrm{v} 2}-2 \widehat{k}_{\mathrm{h} 2}(1-\hat{l}) / \hat{l}, \widehat{k}_{\mathrm{h} 1}=k_{\mathrm{h} 1} / k_{\mathrm{v} 1}$,

$\widehat{k}_{\mathrm{v} 2}=k_{\mathrm{v} 2} / k_{\mathrm{v} 1}, \widehat{k}_{\mathrm{h} 2}=k_{\mathrm{h} 2} / k_{\mathrm{v} 1}, \gamma_{1}=\widehat{k}_{\mathrm{h} 1}\left(1-\widehat{l}^{2}\right) / \widehat{l}^{3}$,

$\gamma_{2}=\widehat{k}_{\mathrm{h} 2}\left(1-\hat{l}^{2}\right) / \widehat{l}^{3}, \mu=m_{2} / m_{1}, \widehat{F}_{\mathrm{e}}=F_{\mathrm{e}} / k_{\mathrm{v} l} l_{1}$,

$\Omega=\omega / \omega_{\mathrm{n}}, \tau=\omega_{n} t, \omega_{\mathrm{n}}=\left(k_{\mathrm{v} 1} / m_{1}\right)^{1 / 2}, \widehat{x}_{1}=x_{1} / l_{1}$,

$\widehat{x}_{2}=x_{2} / l_{2}, \hat{l}=l_{1} / l_{\mathrm{o} 1}=l_{2} / l_{\mathrm{o} 2},(\cdot)^{\prime}=\mathrm{d}(\cdot) / \mathrm{d} \tau$.
The harmonic balance method is used to determine the transmissibility in a similar manner as that for the single stage isolator. The non-dimensional responses of the twostage isolator are assumed to have the form of

$\left\{\begin{array}{l}\widehat{x}_{1}(\tau) \\ \widehat{x}_{2}(\tau)\end{array}\right\}=\left\{\begin{array}{l}\widehat{X}_{1} \cos \left(\Omega \tau+\varphi_{X 1}\right) \\ \widehat{X}_{2} \cos \left(\Omega \tau+\varphi_{X 2}\right)\end{array}\right\}$,

The resulting amplitude-frequency matrix equation is given by

$\left(\widehat{\mathbf{K}}(\widehat{X})-\Omega^{2} \widehat{\mathbf{M}}\right) \widehat{\mathbf{X}} \boldsymbol{\Phi}_{X}+\Omega \widehat{\mathbf{C}}_{v} \widehat{\mathbf{X}} \boldsymbol{\Phi}_{X} \mathbf{A}$
$+\Omega \widehat{\mathbf{C}}(\widehat{X}) \widehat{\mathbf{X}} \mathbf{\Phi}_{X} \mathbf{A}=\widehat{\mathbf{F}} \boldsymbol{\Phi}_{X}$,

where $\widehat{\mathbf{X}}=\left[\begin{array}{cc}\widehat{X_{1}} & 0 \\ 0 & \widehat{X_{2}}\end{array}\right], \mathbf{A}=\left[\begin{array}{cc}0 & -1 \\ 1 & 0\end{array}\right], \widehat{\mathbf{F}}=\left[\begin{array}{cc}\widehat{F}_{\mathrm{e}} & 0 \\ 0 & 0\end{array}\right]$,

$\boldsymbol{\Phi}_{X}=\left[\begin{array}{cc}\cos \varphi_{X 1} & -\sin \varphi_{X 1} \\ \cos \varphi_{X 2} & -\sin \varphi_{X 2}\end{array}\right], \widehat{\mathbf{C}}_{\mathrm{v}}=2\left[\begin{array}{cc}\zeta_{v 1} & -\zeta_{v 1} \\ -\zeta_{v 1} & \zeta_{v 1}+\zeta_{v 2}\end{array}\right]$,

$\widehat{\mathbf{C}}_{\mathrm{n}}(\widehat{X})=$

$2\left[\begin{array}{cc}\frac{1}{4} \zeta_{\mathrm{n} 1}\left(X_{1}^{2}+2 X_{2}^{2}\right) & -\frac{1}{4} \zeta_{\mathrm{n} 1}\left(X_{2}^{2}+2 X_{1}^{2}\right) \\ -\frac{1}{4} \zeta_{\mathrm{n} 1}\left(X_{1}^{2}+2 X_{2}^{2}\right) & \frac{1}{4} \zeta_{\mathrm{n} 1}\left(X_{2}^{2}+2 X_{1}^{2}\right)+\frac{1}{4} \zeta_{\mathrm{n} 2} \bar{X}_{2}^{2}\end{array}\right]$,

$\mathbb{\mathbf { K }}(\bar{X})=$

$\left[\begin{array}{cc}\Omega_{1}{ }^{2}+\frac{3}{4} \gamma_{1}\left(X_{1}^{2}-2 X_{2}^{2}\right) & -\Omega_{1}{ }^{2}+\frac{3}{4} \gamma_{1}\left(-X_{2}^{2}+2 X_{1}^{2}\right) \\ -\Omega_{1}{ }^{2}-\frac{3}{4} \gamma_{1}\left(X_{1}^{2}-2 X_{2}^{2}\right) & \Omega_{1}{ }^{2}+\Omega_{2}{ }^{2}-\frac{3}{4} \gamma_{1}\left(-X_{2}^{2}+2 X_{1}^{2}\right)+\frac{3}{4} \gamma_{2} X_{2}^{2}\end{array}\right]$.

Assuming the non-dimensional force transmitted is given by $\widehat{f_{\mathrm{t}}}(\tau)=\widehat{F_{\mathrm{t}}} \cos \left(\Omega \tau+\varphi_{\mathrm{T}}\right)$, the magnitude of the force can be determined from:

$$
\begin{aligned}
\widehat{F}_{\mathrm{t}} \boldsymbol{\Phi}_{\mathrm{t}}= & \widehat{\mathbf{K}}_{\mathrm{t}}(\widehat{X}) \widehat{\mathbf{X}} \boldsymbol{\Phi}_{F}+\Omega \widehat{\mathbf{C}}_{\mathrm{tv}} \widehat{\mathbf{X}} \boldsymbol{\Phi}_{F} \mathbf{A} \\
& +\Omega \widehat{\mathbf{C}}_{\mathrm{tn}}(\bar{X}) \widehat{\mathbf{X}} \boldsymbol{\Phi}_{F} \mathbf{A},
\end{aligned}
$$

where $\widehat{\mathbf{K}}_{\mathrm{t}}(\widehat{X})=\left[\begin{array}{ll}0 & \widehat{k}_{\mathrm{v} 2}-2 \widehat{k}_{\mathrm{h} 2}(1-\widehat{l})+\widehat{k}_{\mathrm{h} 2} \widehat{X}_{2}^{2} / \widehat{l}\end{array}\right]$,

$\widehat{\mathbf{C}}_{\mathrm{tv}}=2\left[\begin{array}{ll}0 & \zeta_{\mathrm{v} 2}\end{array}\right] \widehat{\mathbf{C}}_{\mathrm{tn}}(\widehat{X})=2\left[\begin{array}{ll}0 & \frac{1}{4} \zeta_{\mathrm{h} 2} \widehat{X}_{2}^{2}\end{array}\right]$,

$\boldsymbol{\Phi}_{\mathrm{t}}=\left[\begin{array}{ll}\cos \varphi_{\mathrm{T}} & \sin \varphi_{\mathrm{T}}\end{array}\right]$.

The force transmissibility is given by $\left|T_{F}\right|=\widehat{F}_{\mathrm{t}} / \widehat{F}_{\mathrm{e}}$, and this can be determined by substituting the forces from eqs. (19) and (20).

\subsection{Numerical validation}

The analytical results for the force transmissibility are approximately derived in this manuscript. The solid lines in Figure 5 plot the force transmissibility of the two-stage HSLDS isolation system with both heavy damping and light damping. The approximate analytical results are compared with the numerical results via direct numerical integration of 


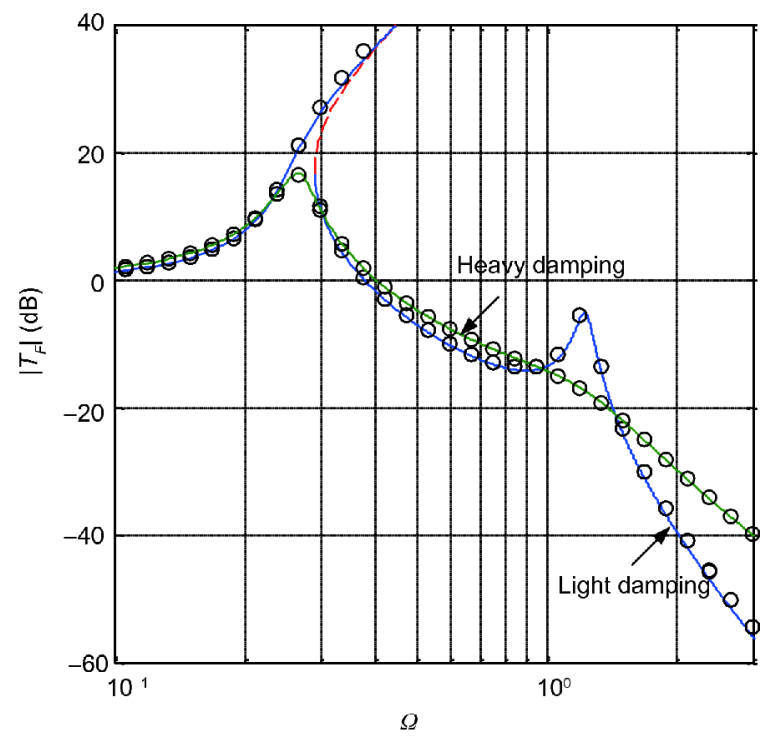

Figure 5 (Color online) Comparison of force transmissibility between HBM and numerical method. The parameters are $\hat{l}=0.7, \widehat{F}_{\mathrm{e}}=0.0022$, $\mu=0.2, \widehat{k}_{\mathrm{v} 2}=1, \widehat{k}_{\mathrm{h} 1}=1, \widehat{k}_{\mathrm{h} 2}=1$. HBM solution: stable solution (solid line), unstable solution (dashed line). Numerical solution: black 'o'. Light damping $\zeta_{\mathrm{v} 1}=\zeta_{\mathrm{v} 2}=0.005, \zeta_{\mathrm{h} 1}=\zeta_{\mathrm{h} 2}=0.005$. Heavy damping $\zeta_{\mathrm{v} 1}=\zeta_{\mathrm{v} 2}=0.5$, $\zeta_{\mathrm{h} 1}=\zeta_{\mathrm{h} 2}=0.5$.

the equation of motion at each frequency of excitation. The ' $\mathrm{o}$ ' in Figure 5 represents the numerical solution for both increasing and decreasing frequency. The analytical and the numerical results perfectly agree in the stable portions for both heavy damping and light damping. To determine whether the method of harmonic balance correctly captures the dynamic behavior of the parameters chosen, the frequency response curves (FRC) of the two masses are plotted in Figure 6(a) and (b) with the numerical results via the RungeKutta scheme [27-31]. The figure shows that there is reasonable agreement, and the method of harmonic balance can be used for further investigation of the dynamic behavior.

\subsection{Analysis of the different parameters}

The force transmissibilities of the two configurations for the two-stage vibration isolators are shown in Figure 7 for an excitation force that is set to the same value as for the singlestage isolator, i.e., $\widehat{F_{\mathrm{e}}}=0.0022$ and $\hat{l}=0.7, \mu=0.2$, $\widehat{k}_{\mathrm{v} 2}=\widehat{k}_{\mathrm{h} 1}=\widehat{k}_{\mathrm{h} 2}=1$. Also shown for comparison is a faint grey line, which is the transmissibility of the system with the horizontal springs and dampers removed, and with $\zeta_{\mathrm{v} 1}=\zeta_{\mathrm{v} 2}=0.005$. Figure 7 (a) illustrates the effects on the force transmissibility for the system that only has vertical damping in both stages. The figure shows that although the damping is effective at reducing the transmitted force at the resonance frequencies, the damping is detrimental to the isolation performance at high frequencies, as discussed in ref. [11]. In addition, Figure 7(b) shows the effects of using only horizontal damping in the two-stage isolator. Similar to the performance of the single-stage isolator, the damping is less-effective at reducing the response at the resonance frequencies, but the damping does not degrade the isolation performance at high frequencies.

The effects of the nonlinear stiffness and mass ratio are investigated when the two-stage isolator only has horizontal damping fixed at $\zeta_{\mathrm{h} 1}=\zeta_{\mathrm{h} 2}=0.05$. Figure 8(a) shows the effect on force transmissibility when nonlinear stiffness is changed by changing of horizontal stiffness in the two-stage isolator. The figure shows that as the peaks of the force transmissibility shift to lower frequencies, the isolation region is extended to lower frequencies. Figure 8(b) shows the variations of the force transmissibility against the mass ratio. In particular, the second peak shifts to lower frequencies, but the first peak remains at almost the same frequency. The
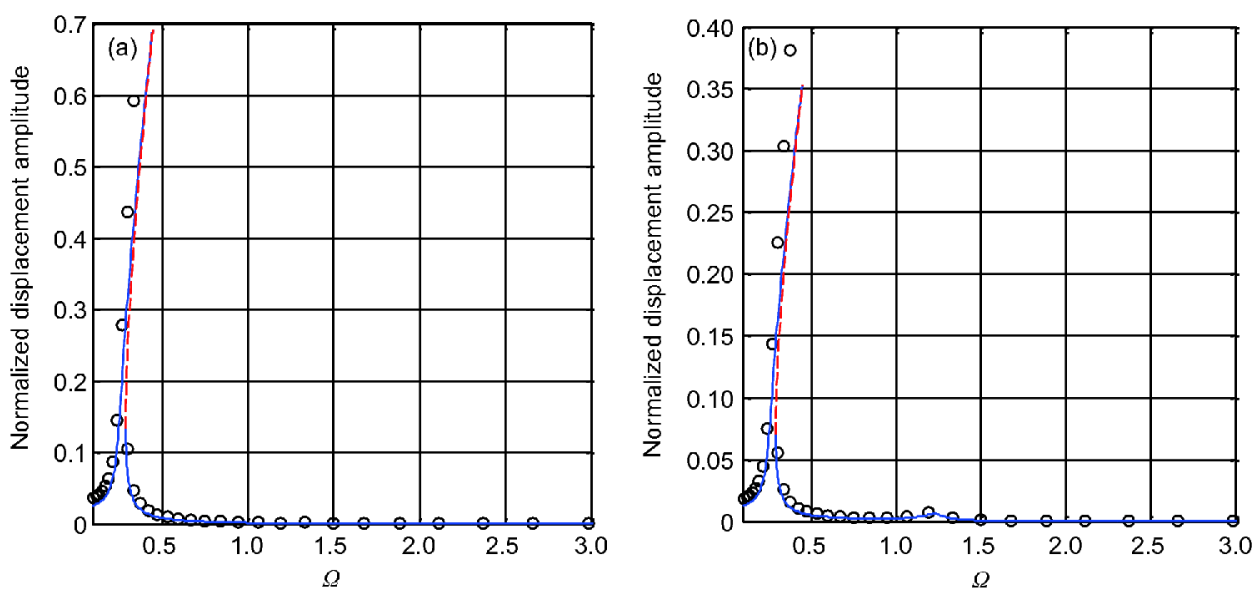

Figure 6 (Color online) The frequency response curve (FRC) of the two masses in the HSLDS isolation system with damping nonlinearity. The parameters are the same as the parameters in Figure 5. (a) FRC of $m_{1}$, (b) FRC of $m_{2}$. HBM solution: stable solution (solid line), unstable solution (dashed line). Numerical solution: black 'o'. 

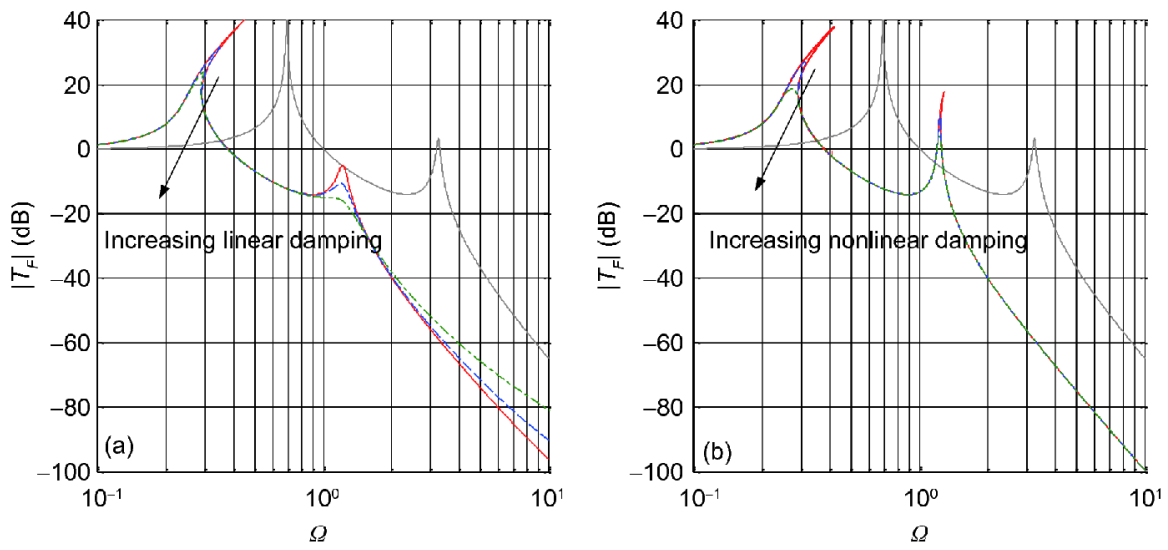

Figure 7 (Color online) Illustration of the effects on the force transmissibility of the two-stage nonlinear isolator if the upper and lower vertical damping $\left(\zeta_{\mathrm{v} 1}, \zeta_{\mathrm{v} 2}\right)$ and the upper and lower horizontal damping $\left(\zeta_{\mathrm{h} 1}, \zeta_{\mathrm{h} 2}\right)$ are changed. The parameters are the same as the parameters in the previous figures: (a) only vertical damping; solid line, $\zeta_{\mathrm{v} 1}=\zeta_{\mathrm{v} 2}=0.005$; dashed line, $\zeta_{\mathrm{v} 1}=\zeta_{\mathrm{v} 2}=0.01$; and dashed-dotted line, $\zeta_{\mathrm{v} 1}=\zeta_{\mathrm{v} 2}=0.02$; (b) only horizontal damping; solid line, $\zeta_{\mathrm{h} 1}=\zeta_{\mathrm{h} 2}=0.05$; dashed line, $\zeta_{\mathrm{h} 1}=\zeta_{\mathrm{h} 2}=0.5$; and dashed-dotted line, $\zeta_{\mathrm{h} 1}=\zeta_{\mathrm{h} 2}=1$. The faint grey line represents a linear isolator that only has vertical stiffness and damping with $\zeta_{\mathrm{v} 1}=\zeta_{\mathrm{v} 2}=0.005$.
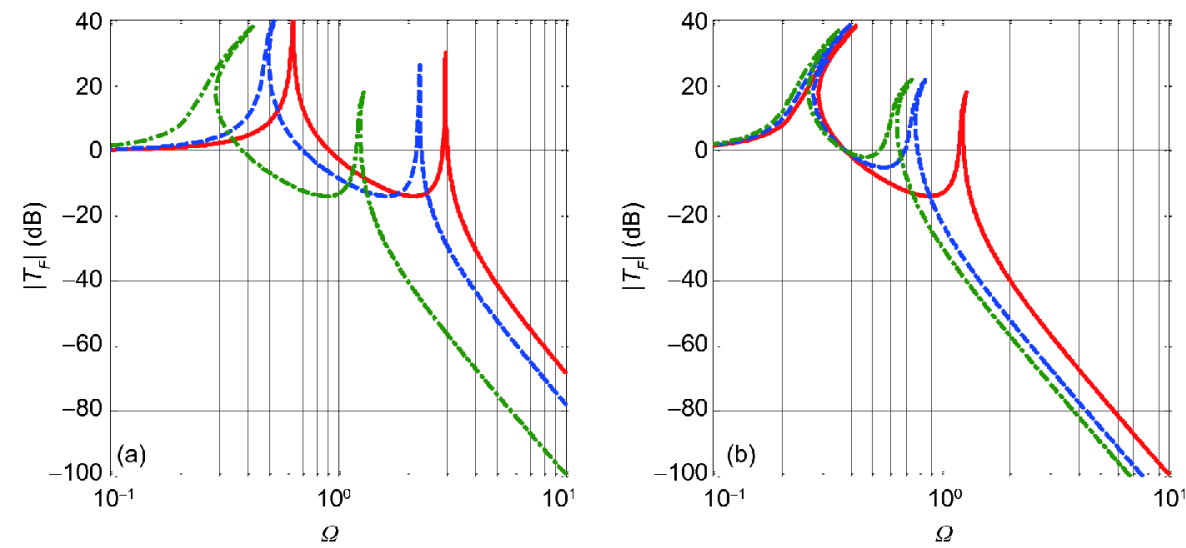

Figure 8 (Color online) Comparison of the force transmissibility of the two-stage nonlinear isolator with different nonlinear stiffness or the mass ratio, and the vertical damping and horizontal damping are fixed at $\zeta_{\mathrm{v} 1}=\zeta_{\mathrm{v} 2}=0$. The parameters are the same as the parameters in the previous figures, $\zeta_{\mathrm{h} 1}=\zeta_{\mathrm{h} 2}=0.05$. (a) Effects of the nonlinear stiffness $\left(\widehat{k}_{\mathrm{h} 1}, \widehat{k}_{\mathrm{h} 2}\right)$. Solid line, $\widehat{k}_{\mathrm{h} 1}=\widehat{k}_{\mathrm{h} 2}=0.2$; dashed line, $\widehat{k}_{\mathrm{h} 1}=\widehat{k}_{\mathrm{h} 2}=0.6$; and dashed-dotted line, $\widehat{k}_{\mathrm{h} 1}=\widehat{k}_{\mathrm{h} 2}=1$. (b) Effects of the mass ratio $\mu$. Solid line, $\mu=0.2$; dashed line, $\mu=0.6$; and dashed-dotted line, $\mu=1$.

magnitude of force transmissibility is significantly reduced at high frequencies.

\section{Conclusions}

This article shows the effects of using nonlinear stiffness and damping in a single-stage and a two-stage vibration isolator. The type of stiffness was of the HSLDS type, and all nonlinearities were introduced by the geometrical configuration. The dampers were either aligned with the direction of excitation or orthogonal to the direction of excitation. The results show the HSLDS was advantageous in all cases, allowing the frequency range of isolation to be extended to low frequencies without compromising the static stiffness. Concerning the damping elements, the linear viscous damping which was aligned in the direction of the force excitation had good performance at the resonance frequencies, but the linear viscous damping was detrimental to the isolation performance at high frequencies. This contrasted with the dampers that were aligned orthogonally to the direction of the force excitation, because they performed well at high frequencies.

This work was supported by the State Key Program of National Natural Science of China (Grant No. 11232009), the National Natural Science Foundation of China (Grant Nos. 11502135 \& 11572182) and the Innovation Program of Shanghai Municipal Education Commission (Grant No. 2017-01-07-00-09-E00019).

$1 \mathrm{Lu} \mathrm{Z} \mathrm{Q}$, Chen L Q. Some recent progresses in nonlinear passive isolations of vibrations. Chin J Theor Appl Mech, 2017, 49: 550-564

2 Kovacic I, Brennan M J, Waters T P. A study of a nonlinear vibration 
isolator with a quasi-zero stiffness characteristic. J Sound Vib, 2008, 315: 700-711

3 Carrella A, Brennan M J, Kovacic I, et al. On the force transmissibility of a vibration isolator with quasi-zero-stiffness. J Sound Vib, 2009, 322: 707-717

4 Carrella A, Brennan M J, Waters T P, et al. Force and displacement transmissibility of a nonlinear isolator with high-static-low-dynamicstiffness. Int J Mech Sci, 2012, 55: 22-29

5 Zhang W, Zhao J B. Analysis on nonlinear stiffness and vibration isolation performance of scissor-like structure with full types. Nonlinear Dyn, 2016, 86: 17-36

6 Sun $\mathrm{X} \mathrm{T}$, Jing X J, Xu J, et al. Vibration isolation via a scissor-like structured platform. J Sound Vib, 2014, 333: 2404-2420

$7 \mathrm{Xu}$ J, Sun X. A multi-directional vibration isolator based on QuasiZero-Stiffness structure and time-delayed active control. Int $\mathrm{J}$ Mech Sci, 2015, 100: 126-135

8 Sun X T, Jing X J, Cheng L, et al. A 3-D quasi-zero-stiffness-based sensor system for absolute motion measurement and application in active vibration control. IEEE/ASME Trans Mechatron, 2015, 20: 254-262

9 Zhou J X, Xiao Q Y, Xu D L, et al. A novel quasi-zero-stiffness strut and its applications in six-degree-of-freedom vibration isolation platform. J Sound Vib, 2017, 394: 59-74

$10 \mathrm{Xu}$ D L, Yu Q P, Zhou J X, et al. Theoretical and experimental analyses of a nonlinear magnetic vibration isolator with quasi-zerostiffness characteristic. J Sound Vib, 2013, 332: 3377-3389

11 Lu Z Q, Yang T J, Brennan M J, et al. On the performance of a twostage vibration isolation system which has geometrically nonlinear stiffness. J Vib Acoust, 2014, 136: 064501

12 Lu Z Q, Yang T J, Brennan M J, et al. Experimental investigation of a two-stage nonlinear vibration isolation system with high-static-lowdynamic stiffness. J Appl Mech, 2017, 84: 021001

$13 \mathrm{Xu} \mathrm{Z} \mathrm{D,} \mathrm{Zeng} \mathrm{X,} \mathrm{Huang} \mathrm{X} \mathrm{H,} \mathrm{et} \mathrm{al.} \mathrm{Experimental} \mathrm{and} \mathrm{numerical}$ studies on new multi-dimensional earthquake isolation and mitigation device: Horizontal properties. Sci China Tech Sci, 2010, 53: 2658 2667

14 Li H, Liu D, Jiang L, et al. Self-synchronization theory of dual motor driven vibration system with two-stage vibration isolation frame. Appl Math Mech-Engl Ed, 2015, 36: 265-278

15 Ravindra B, Mallik A K. Hard duffing-type vibration isolator with combined Coulomb and viscous damping. Int J Non-Linear Mech, 1993, 28: 427-440

16 Ravindra B, Mallik A K. Stability analysis of a non-linearly damped duffing oscillator. J Sound Vib, 1994, 171: 708-716

17 Ravindra B, Mallik A K. Chaotic response of a harmonically excited mass on an isolator with non-linear stiffness and damping characteristics. J Sound Vib, 1995, 182: 345-353

18 Ravindra B, Mallik A K. Role of nonlinear dissipation in soft Duffing oscillators. Phys Rev E, 1994, 49: 4950-4954

19 Peng Z K, Meng G, Lang Z Q, et al. Study of the effects of cubic nonlinear damping on vibration isolations using Harmonic Balance Method. Int J Non-Linear Mech, 2012, 47: 1073-1080

20 Peng Z K, Lang Z Q, Meng G, et al. Reducing force transmissibility in multiple degrees of freedom structures through anti-symmetric nonlinear viscous damping. Acta Mech Sin, 2012, 28: 1436-1448

21 Lang Z Q, Jing X J, Billings S A, et al. Theoretical study of the effects of nonlinear viscous damping on vibration isolation of SDOF systems. J Sound Vib, 2009, 323: 352-365

22 Xiao Z, Jing X J, Cheng L. The transmissibility of vibration isolators with cubic nonlinear damping under both force and base excitations. J Sound Vib, 2013, 332: 1335-1354

23 Ho C, Lang Z Q, Billings S A. Design of vibration isolators by exploiting the beneficial effects of stiffness and damping nonlinearities. J Sound Vib, 2014, 333: 2489-2504

24 Tang B, Brennan M J. A comparison of two nonlinear damping mechanisms in a vibration isolator. J Sound Vib, 2013, 332: 510-520

25 Carranza J C, Brennan M J, Tang B. Sources and propagation of nonlinearity in a vibration isolator with geometrically nonlinear damping. J Vib Acoust, 2016, 138: 024501

26 Li H L, Chen Y S, Hou L, et al. Periodic response analysis of a misaligned rotor system by harmonic balance method with alternating frequency/time domain technique. Sci China Tech Sci, 2016, 59: $1717-1729$

27 Yan Q Y, Ding H, Chen L Q. Nonlinear dynamics of axially moving viscoelastic Timoshenko beam under parametric and external excitations. Appl Math Mech-Engl Ed, 2015, 36: 971-984

28 Jiang W A, Chen L Q. A piezoelectric energy harvester based on internal resonance. Acta Mech Sin, 2015, 31: 223-228

29 Ding H. Steady-state responses of a belt-drive dynamical system under dual excitations. Acta Mech Sin, 2016, 32: 156-169

30 Ding H, Huang L L, Mao X Y, et al. Primary resonance of traveling viscoelastic beam under internal resonance. Appl Math Mech-Engl Ed, 2017, 38: 1-14

31 Li X, Zhang Y W, Ding H, et al. Integration of a nonlinear energy sink and a piezoelectric energy harvester. Appl Math Mech-Engl Ed, 2017, 38: $1019-1030$ 